\title{
Efeito da adição de polietilenoglicóis nas formulações de microesferas de acetobutirato de celulose sobre a eficiência de encapsulação da carbamazepina e morfologia das partículas
}

\author{
Betina Giehl Zanetti ${ }^{1}$, Valdir Soldi ${ }^{2}$, Elenara Lemos-Senna ${ }^{1 *}$ \\ ${ }^{1}$ Centro de Ciências da Saúde, Departamento de Ciências Farmacêuticas, Universidade Federal de Santa Catarina, \\ ${ }^{2}$ Laboratório de Polímeros, Departamento de Química, Universidade Federal de Santa Catarina
}

*Correspondência:

E. Lemos-Senna

Universidade Federal de Santa

Catarina

Centro de Ciências da Saúde

Campus Trindade

88040-900 - Florianópolis - Brasil

E-mail: lemos@ccs.ufsc.br
O desenvolvimento de microesferas de carbamazepina (CBZ) de liberação prolongada tem sido realizado, empregando-se a técnica de emulsificação/evaporação do solvente e o acetobutirato de celulose (ABC70) para a obtenção da matriz polimérica. Buscando modular a velocidade de liberação do fármaco, a adição de polietilenoglicóis na fase interna da emulsão foi testada. Quando comparados com as microesferas preparadas unicamente com ABC70, o teor e a eficiência de encapsulação da CBZ, estimados após análise por CLAE, foram drasticamente reduzidos com a adição de PEG4000. Entretanto, a adição de PEG 1500 perfazendo $10 \%$ da massa total de polímero não pareceu alterar o teor de $C B Z$, demonstrando que o peso molecular do PEG influencia a encapsulação da CBZ. Partículas mais porosas foram visualizadas por MEV, quando o PEG1500 ou 4000 foram adicionados. Entretanto, a perda da forma esférica foi observada quando uma mistura de ABC70:PEG 4000 1:1 foi empregada. A adição de polietilenoglicóis não pareceu afetar o diâmetro médio das microesferas. A redução na encapsulação da CBZ foi associada à extração do PEG durante a etapa de evaporação do solvente e a sua capacidade de dissolvê-la na fase externa da emulsão. $O$ controle do desenvolvimento da presença de poros, pelo ajuste da concentração e do peso molecular do PEG, pode ser útil na modulação do perfil de liberação do fármaco.
Unitermos:

- Carbamazepina

- Microesferas

- Acetobutirato de celulose

- Polietilenoglicol 1500

- Polietilenoglicol 4000

- Emulsificação

- Evaporação do solvente

\section{INTRODUÇÃO}

A carbamazepina (CBZ) é um fármaco anticonvulsivante amplamente empregado no tratamento da epilepsia, eficaz contra crises generalizadas tônico-clônicas e parciais. Quimicamente, trata-se de um derivado do iminoestilbeno, relacionado com os antidepressivos tricíclicos, cujo mecanismo de ação parece envolver a limitação das crises epiléticas pela redução da potenciação pós-tetânica da transmissão sináptica. Embora na fase inicial do seu tratamento a CBZ apresente um $\mathrm{t}^{1} / 2$ longo, este é reduzido com o uso crônico, fazendo com que o fármaco seja administrado em 3 ou 4 doses diárias (Reynolds, 1989; Korolkovas, 1998). Apesar de completa, a absorção deste fármaco após administração oral é lenta e irregular, conduzindo a ampla variabilidade nas concentrações séricas e ao aparecimento 
de efeitos adversos, tais como náuseas, vômitos, visão turva, sonolência, ataxia e vertigem (USP DI, 1997; Korolkovas, 1998). Neste sentido, a adesão do paciente estaria relacionada não somente à simplificação do regime de administração, mas igualmente à aceitação da própria medicação em termos de um perfil favorável de tolerabilidade. Há, atualmente, quantidade suficiente de evidências de que nestes aspectos, as preparações de liberação prolongada apresentam vantagens práticas sobre as formulações convencionais, tais como a redução do número de doses diárias do medicamento, a redução dos efeitos colaterais e a manutenção da concentração plasmática em níveis terapêuticos.

A microencapsulação tem-se mostrado uma excelente estratégia no desenvolvimento de formulações de liberação prolongada. Dentre as técnicas de microencapsulação destaca-se a emulsificação/evaporação do solvente, amplamente utilizada face à simplicidade dos procedimentos envolvidos e às possibilidades de otimização de características físicas e fisico-químicas das mesmas. A estrutura final e a composição das microesferas é resultante de uma complexa contribuição das características do polímero, fármaco, solventes e agentes emulsificantes (Watts, 1990).

A associação de polímeros em sistemas matriciais tem-se mostrado recentemente como importante estratégia farmacotécnica na busca da modulação e do prolongamento da liberação do fármaco. Em especial, os polietilenoglicóis (PEG) têm sido empregados com este objetivo. Estes adjuvantes farmacêuticos são amplamente utilizados em formulações destinadas às vias parenteral, tópica, oral e retal, como plastificantes, co-solventes ou ainda na preparação de dispersões sólidas (Wade, Weller, 1994). Em associação com outros polímeros na elaboração de sistemas de liberação matriciais, tais como as micropartículas, os polietilenoglicóis podem conduzir à formação de estruturas mais porosas, tornando-se útil na melhoria da velocidade de liberação do fármaco (Yeh et al., 1995; Khidr et al., 1998; Yeh, 2000).

$\mathrm{O}$ acetobutirato de celulose (ABC) é um polímero insolúvel em água, disponível em vários graus de viscosidade, em função do seu peso molecular. Este polímero tem sido empregado na obtenção de matrizes de liberação prolongada preparadas pela técnica de compressão direta, bem como na obtenção de membranas semi-permeáveis dos sistemas de bomba osmótica (Eastman, 2000; Yuan, Wu, 2001). A sua aplicação na preparação de microesferas pela técnica de emulsificação/evaporação do solvente tem sido intensamente investigada (Bhardwaj et al., 1995; Khawla et al., 1996; Arnaud et al.; 1996, Khird et al., 1998). Entretanto, estes polímeros tendem a formar estruturas rígidas e compactas, cuja permeabilidade pode ser alterada pela adição de polietilenoglicóis.

Neste trabalho, a viabilidade da preparação de microesferas pela técnica de emulsificação/evaporação do solvente, a partir de blendas de ABC70 e polietilenoglicóis, foi verificada com vistas à obtenção de sistemas que permitam a modulação da velocidade de liberação da carbamazepina. Para este fim, a avaliação da influência da adição de polietilenoglicóis na fase interna da emulsão sobre a eficiência de encapsulação da carbamazepina e sobre a morfologia das partículas foi realizada.

\section{MATERIAL E MÉTODOS}

\section{Material}

A carbamazepina foi adquirida da Galena Química e Farmacêutica (Brasil). O acetobutirato de celulose (PM $70.000 ; 37 \%$ de grupamentos butirila e $13,5 \%$ de grupamentos acetila) foi adquirido da Aldrich Chemical Co. (EUA), o monoleato de sorbitano (SPAN 80), da Beraca (Brasil), o óleo mineral, da Importadora Química Delaware (Brasil), o PEG1500 e 4000, da Henrifarma (Brasil). Todos os outros solventes empregados, com exceção da acetonitrila para CLAE, possuíam grau de pureza pro analysis (p.a).

\section{Métodos}

\section{Preparação das microesferas}

As microesferas foram preparadas pelo método de emulsificação/evaporação do solvente descrita na literatura por Watts et al. (1990), conforme demonstrado na Figura 1. A técnica consistiu em emulsificar, sob forma de finas gotículas, $20 \mathrm{~mL}$ de uma solução de acetobutirato de celulose PM 70000 (ABC70) e carbamazepina (CBZ) em acetona (fase interna) em $100 \mathrm{~mL}$ de vaselina líquida contendo $1 \%(\mathrm{p} / \mathrm{v})$ de monoleato de sorbitano (SPAN 80 ) como estabilizante (fase externa). A vaselina líquida foi previamente saturada com a acetona e, quando necessário, polietilenoglicol 1500 ou 4000 (PEG 1500 ou 4000) foi adicionado à fase interna antes do procedimento de emulsificação. A emulsão formada foi mantida sob agitação de $650 \mathrm{rpm}$, durante 24 horas, à temperatura ambiente, até a completa evaporação do solvente volátil da fase interna. As partículas formadas foram filtradas, lavadas três vezes com $50 \mathrm{~mL}$ de $n$-hexano e secas em estufa a vácuo, durante 4 horas, à temperatura ambiente. A concentração total de polímero (ABC ou mistura de $\mathrm{ABC}$ e $\mathrm{PEG)}$ na fase interna e as relações fármaco:polímero foram mantidas em $2,5 \%(\mathrm{p} / \mathrm{v})$ e $1: 2$, respectivamente. Cinco 


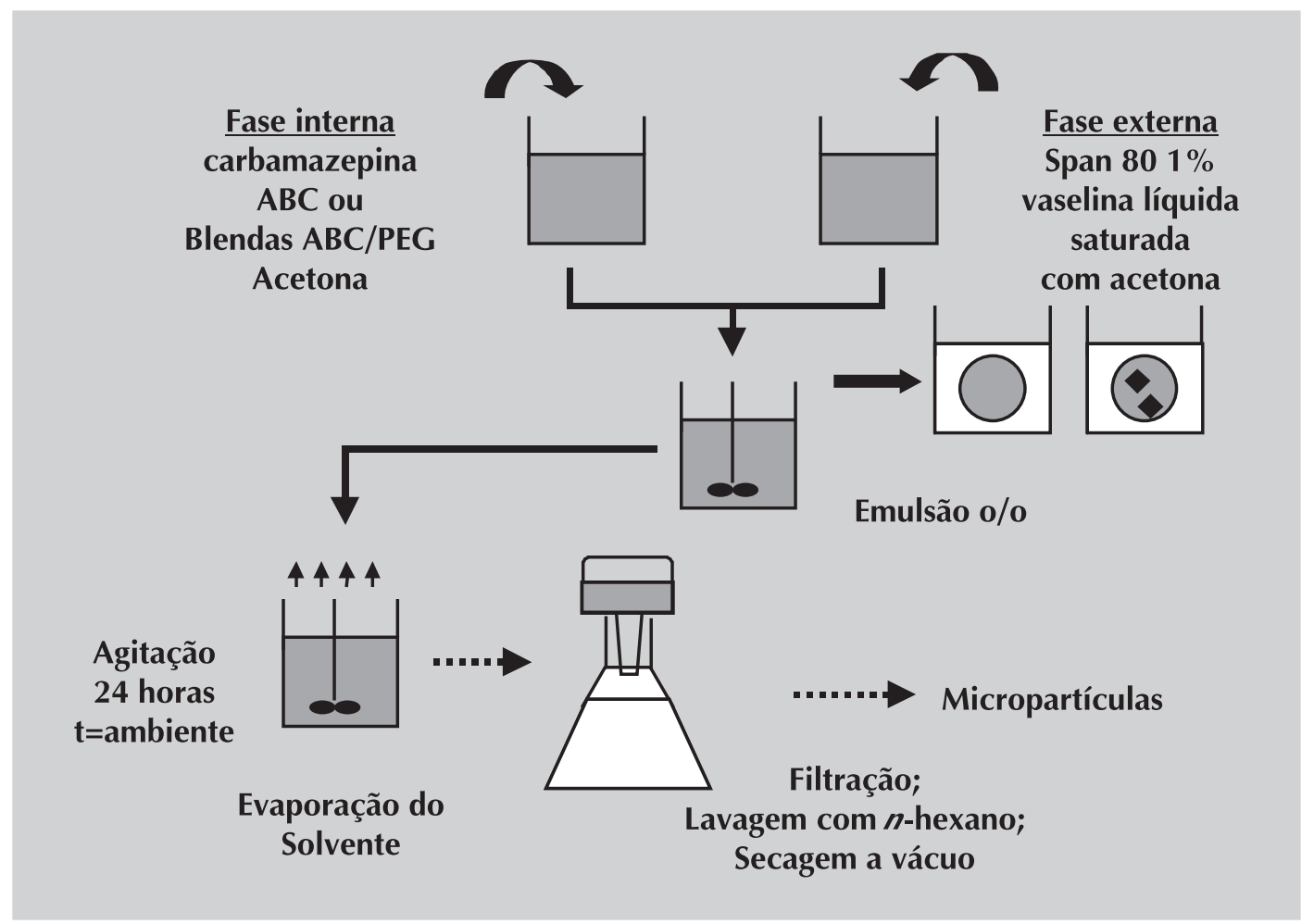

FIGURA 1 - Esquema de preparação das microesferas.

formulações foram testadas tendo-se como variável a adição de PEG 1500 ou 4000 (Tabela I).

\section{Caracterização das microesferas}

Determinação da eficiência de encapsulação e do teor de carbamazepina

A eficiência de encapsulação e o teor de fármaco foram estimados após determinação, por cromatografia líquida de alta eficiência (CLAE), da concentração de CBZ nas soluções obtidas após completa dissolução das partículas com acetonitrila. As condições de análise empregadas foram as seguintes: fase móvel, água:acetonitrila (55:45, v/v), coluna, Supelcosil LC-18 (250 mm x 4,6 mm i.d.; Supelco, EUA); volume de injeção, $20 \mu \mathrm{L}$; fluxo $1,5 \mathrm{~mL} / \mathrm{min}$ e detecção no ultravioleta, $283 \mathrm{~nm}$. As amostras foram injetadas em triplicata e a concentração de CBZ das soluções foi determinada comparando-se as áreas dos picos obtidos com as amostras com aquele obtido a partir

TABELA I - Formulações de microesferas contendo a carbamazepina

\begin{tabular}{|c|c|c|c|c|c|}
\hline Formulação & 1 & 2 & 3 & 4 & 5 \\
\hline \multicolumn{6}{|l|}{ Fase interna } \\
\hline Carbamazepina (mg) & 250 & 250 & 250 & 250 & 250 \\
\hline Acetobutirato de celulose (mg) & 500 & 450 & 450 & 375 & 250 \\
\hline Polietilenoglicol 1500 (mg) & & 50 & & & \\
\hline Polietilenoglicol 4000 (mg) & & & 50 & 125 & 250 \\
\hline Acetona $(\mathrm{mL}) \mathrm{qsp}$ & 20 & 20 & 20 & 20 & 20 \\
\hline \multicolumn{6}{|l|}{ Fase externa } \\
\hline SPAN $80(g)$ & 1 & 1 & 1 & 1 & 1 \\
\hline Vaselina líquida (mL) & 100 & 100 & 100 & 100 & 100 \\
\hline Relação ABC:PEG & $1: 0$ & $9: 1$ & $9: 1$ & $3: 1$ & $1: 1$ \\
\hline
\end{tabular}


de uma solução padrão de CBZ de concentração conhecida analisada nas mesmas condições. A linearidade do método foi previamente avaliada pela construção de uma curva de calibração na faixa de concentração de CBZ entre 10 e $100 \mu \mathrm{g} / \mathrm{mL}\left(\mathrm{r}^{2}=0,9996\right)$. A eficiência de encapsulação (\%) foi calculada relacionando-se a quantidade de fármaco inicialmente adicionada a formulação e a quantidade obtida nas microesferas. $\mathrm{O}$ teor de fármaco foi estimado como sendo o peso percentual $(\% ; \mathrm{p} / \mathrm{p})$ de CBZ encontrado nas partículas.

\section{Avaliação da morfologia}

A morfologia bem como as propriedades de superfície foram avaliadas pela observação das partículas visualizadas nas fotomicrografias obtidas por microscopia eletrônica de varredura (MEV, Phillips XL-30) após o recobrimento das amostras com uma camada de $350 \AA$ de ouro, em aumentos de 20 e 180 vezes.

\section{Análise granulométrica}

O diâmetro médio das partículas e a distribuição granulométrica foram determinados por meio de medição do diâmetro de Ferret das partículas visualizadas nas fotomicrografias ampliadas obtidas por MEV.

\section{RESULTADOS E DISCUSSÃO}

A preparação das microesferas foi realizada pela técnica que envolve a emulsificação de um solvente orgânico volátil contendo os polímeros e o fármaco (fase interna), em um solvente não volátil (fase externa), seguido da evaporação do primeiro. Após a eliminação do solvente da fase interna, em um processo que implica sua prévia difusão para a fase externa e posterior evaporação na interface líquido-ar, as partículas são formadas e recuperadas por filtração. A escolha dos solventes de ambas as fases da emulsão reside na capacidade dos mesmos em solubilizar o fármaco e polímeros, de modo a obter micropartículas com elevados percentuais de encapsulação. Para a preparação de microesferas a partir de ésteres de celulose, a acetona tem sido freqüentemente empregada como fase interna, sendo esta emulsificada em vaselina líquida previamente saturada com o mesmo solvente. A saturação do solvente da fase externa impede a rápida difusão da acetona, evitando precipitações do polímero, além de favorecer a encapsulação do fármaco (Watts, 1990). Uma vez que a água não é utilizada, a emulsão formada pode ser classificada como sendo óleo em óleo $(\mathrm{O} / \mathrm{O})$, sendo estabilizada por tensoativo de baixo valor de EHL, tal como o monoleato do sorbitano (SPAN 80, EHL $=4,3$ ).

Com o objetivo de avaliar a influência do peso molecular e da concentração de polietilenoglicol sobre as características físico-químicas das microesferas, misturas de ABC70 e PEG 4000 em diferentes proporções (9:1; $3: 1 ; 1: 1)$ foram testadas, mantendo-se em todos os casos a mesma concentração total de polímero na fase interna da emulsão. Da mesma maneira, a adição de PEG 1500 foi testada, mas em uma única concentração (9:1). A sua característica semi-sólida impediria a obtenção de partículas compactas, se adicionado em maiores proporções na mistura. Os valores de eficiência de encapsulação e de teor de carbamazepina obtidos nas condições de preparação empregadas encontram-se demonstradas na Tabela II.

Em comparação com a formulação contendo unicamente ABC70, a adição de PEG 4000 na fase interna da emulsão provocou a redução drástica do conteúdo de fármaco nas partículas. Esta redução atingiu valores superiores a $50 \%$, quando misturas de $\mathrm{ABC} 70$ e PEG $4000 \mathrm{em}$ proporções de 3:1 e 1:1 foram empregadas. Por outro lado, os resultados obtidos indicam que o peso molecular do PEG afeta a eficiência de encapsulação e o teor de carbamazepina nas microesferas. A adição de PEG 1500,

TABELA II - Valores de eficiência de encapsulação, teor de CBZ e diâmetro médio das partículas obtidos para as formulações testadas

\begin{tabular}{ccccc}
\hline Formulação & ABC:PEG & $\begin{array}{c}\text { Teor de CBZ } \\
(\%, \mathrm{p} / \mathrm{p})\end{array}$ & $\begin{array}{c}\text { Eficiência de } \\
\text { encapsulação }(\%)\end{array}$ & $\begin{array}{c}\text { Diâmetro de } \\
\text { partícula }(\mathrm{mm})\end{array}$ \\
\hline 1 & $1: 0$ & $21,02 \pm 0,07$ & $63,13 \pm 0,18$ & $611,48 \pm 112,19$ \\
2 & $9: 1^{\mathrm{a}}$ & $23,86 \pm 0,56$ & $71,67 \pm 1,64$ & $679,99 \pm 114,50$ \\
3 & $9: 1^{\mathrm{b}}$ & $14,20 \pm 0,04$ & $42,95 \pm 0,10$ & nd \\
4 & $3: 1^{\mathrm{b}}$ & $9,48 \pm 0,10$ & $28,46 \pm 0,31$ & $593,01 \pm 113,47$ \\
5 & $1: 1^{\mathrm{b}}$ & $10,13 \pm 0,31$ & $30,46 \pm 0,93$ & $572,51 \pm 102,43$ \\
\hline
\end{tabular}

a PEG 1500; ${ }^{\text {b }}$ PEG 4000; nd = não determinado. 
perfazendo $10 \%$ da massa total de polímero na formulação, não pareceu alterar o teor de CBZ, enquanto a adição de PEG 4000 na mesma proporção conduziu à redução de aproximadamente $30 \%$ na encapsulação deste fármaco.

$\mathrm{Na}$ técnica de emulsificação-evaporação do solvente, o diâmetro médio final das partículas depende, em grande extensão, da velocidade de agitação e do tipo de equipamento empregado. Outras variáveis geralmente estudadas incluem o tipo e a concentração do emulgente, a proporção de volume da fase dispersa em relação à fase contínua, a concentração do polímero e a relação fármaco:polímero na fase interna da emulsão (Watts, 1990; Bahl, 2000). Todas estas variáveis foram mantidas constantes para permitir a avaliação do efeito da adição do polietilenoglicol na eficiência de encapsulação da CBZ e na morfologia das microesferas. A velocidade de agitação foi de $650 \mathrm{rpm}$ para todas as formulações testadas. A mudança na proporção e no peso molecular do PEG adicionado às formulações não pareceu afetar o diâmetro médio das partículas que se manteve em torno de $600 \mu \mathrm{m}$ (Tabela II).

A avaliação da morfologia foi realizada pela observação das microesferas por microscopia eletrônica de varredura (MEV) (Figuras 2 e 3). Partículas esféricas de superfície lisa e compacta foram visualizadas nas fotomicrografias obtidas a partir das microesferas preparadas unicamente com ABC70 (Figura 2). Em contrapartida, a obtenção de estruturas mais rugosas e porosas foi demonstrada quando misturas de ABC70:PEG 1500 (9:1) e ABC70:PEG 4000 (3:1) foram empregadas nas formulações (Figuras 3a e 3b, respectivamente). Entretanto, não somente a presença de grandes poros mas igualmente a perda da forma esférica foram observados nas microesferas preparadas com a mistura de ABC70:PEG 4000 (1:1)(Figura $3 \mathrm{c}$ ). Estes resultados parecem estar relacionados com a não permanência do polietilenoglicol na estrutura final das partículas. De fato, a completa solubilização do polímero na fase externa da emulsão foi verificada em um ensaio, em que somente o PEG foi adicionado à formulação, indicando que a extração do mesmo ocorre durante a etapa de evaporação do solvente. A extração do PEG foi, portanto, responsável pela formação de poros na matriz polimérica.

Da mesma maneira, a redução dos valores de eficiência de encapsulação e de teor de fármaco, visualizados na Tabela II, parece estar relacionada com a eliminação do polietilenoglicol da estrutura final das microesferas. Estes polímeros atuariam como co-solventes, aumentando a solubilidade da CBZ e contribuindo, conseqüentemente, para a partição do fármaco em favor da fase externa. Neste caso, ambos aumento do peso molecular e aumento da concentração de PEG 4000 conduziram ao aumento da
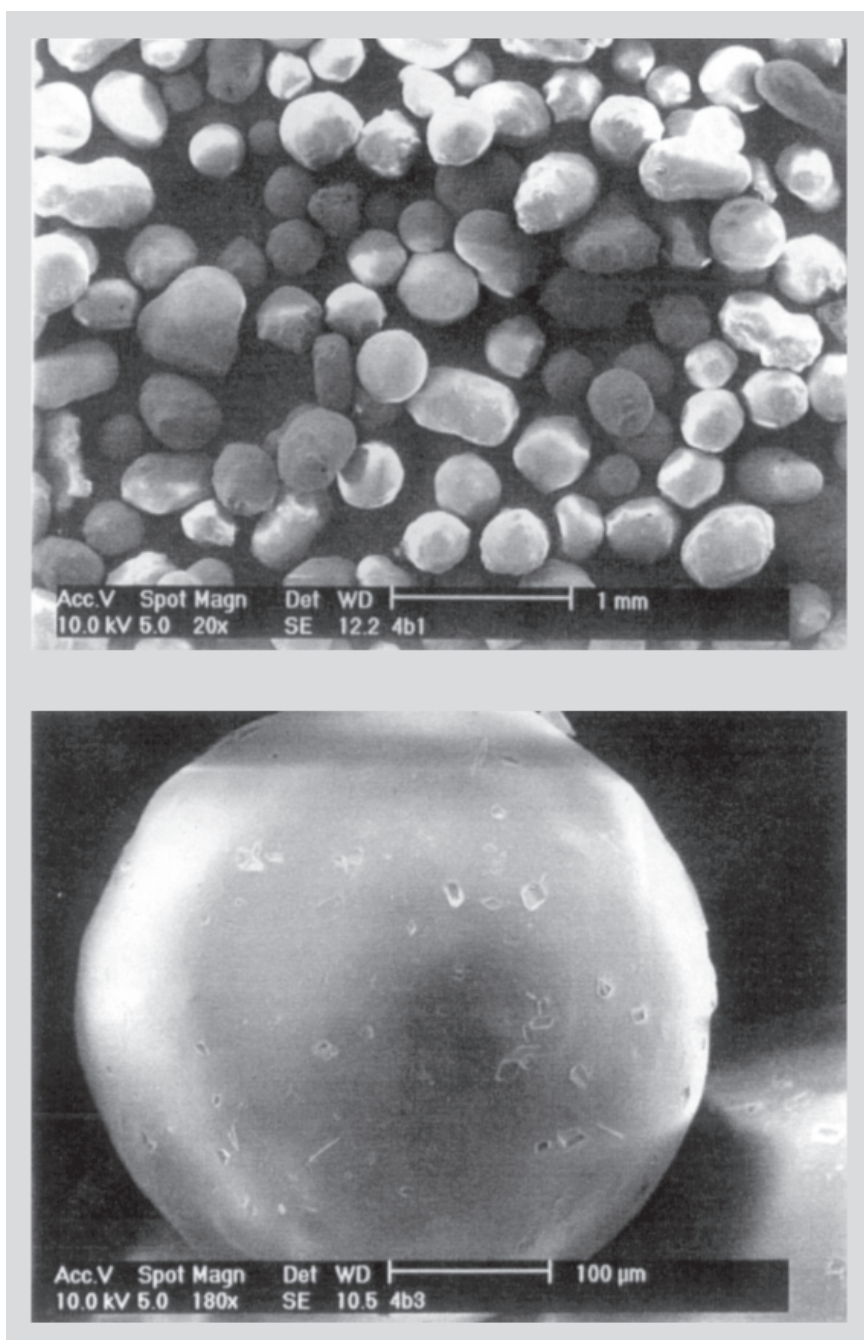

FIGURA 2 - Fotomicrografias obtidas por MEV das microesferas preparadas a partir de $\mathrm{ABC} 70 \mathrm{em}$ aumentos de 20 e 180 vezes.

concentração de CBZ dissolvida na fase externa da emulsão. A fração de carbamazepina não encapsulada foi eliminada após filtração e lavagem das microesferas, explicando a diferença dos valores obtidos. Resultados semelhantes foram relatados por Doshi et al. (1997) e Zerrouk et al. (2001) em estudos de formulação de dispersões sólidas de CBZ e polietilenoglicóis. Este último descreve a formação de complexos solúveis entre fármaco e polímero como sendo o mecanismo responsável pela solubilização da CBZ em água, tendo sido verificada a linearidade entre o aumento da quantidade de fármaco dissolvido e a concentração do polietilenoglicol.

Um breve ensaio de dissolução foi realizado com as partículas preparadas apenas com $\mathrm{ABC} 70$ e com aquelas obtidas com o uso da mistura ABC70/PEG 1500 (9:1), 

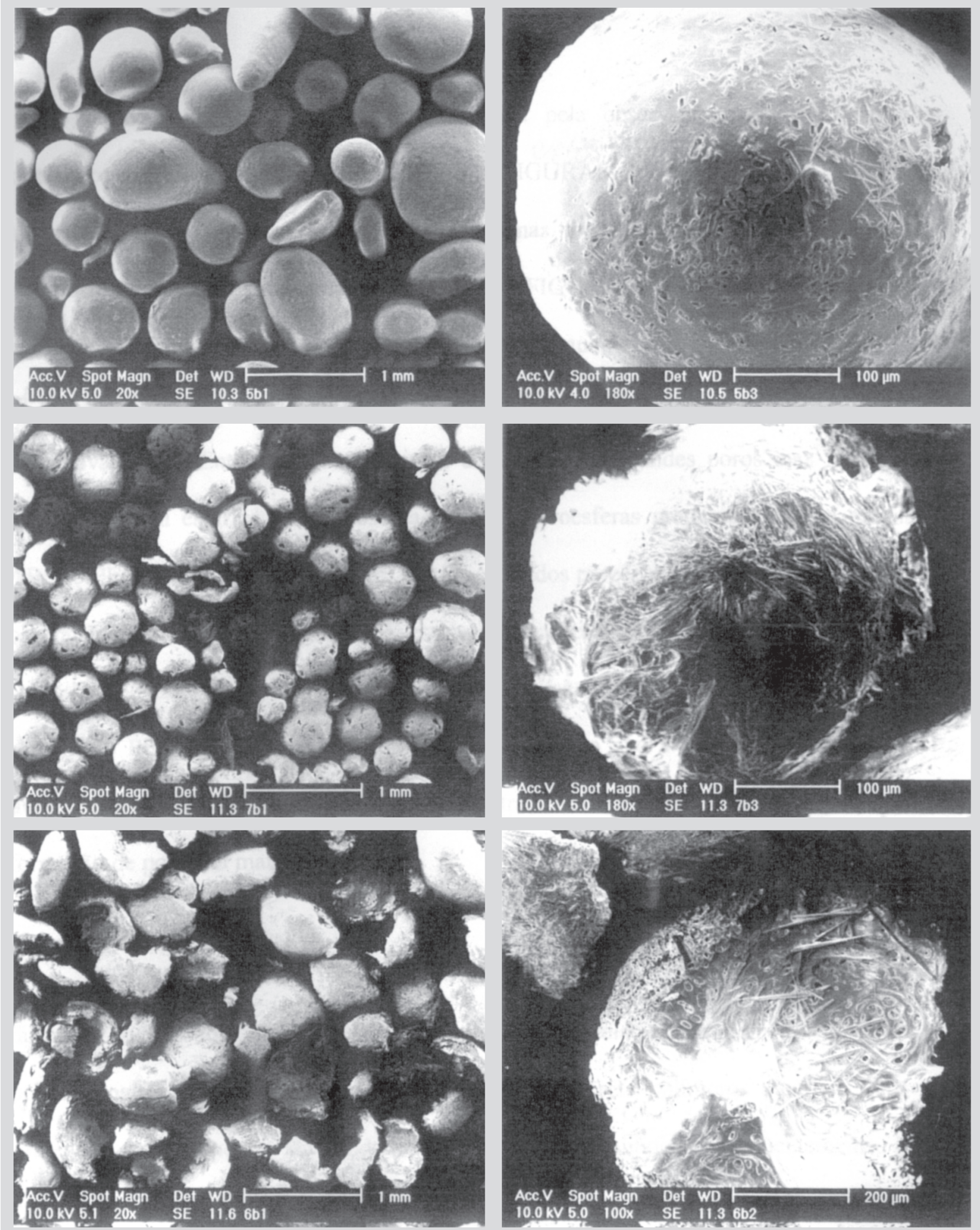

FIGURA 3 - Fotomicrografias obtidas por MEV das microesferas preparadas com misturas de acetobutirato de celulose e polietilenoglicol em aumentos de 20 e 180 vezes. (a) ABC70:PEG 1500 9:1; (b) ABC70:PEG 4000 3:1 e (c) ABC70:PEG 4000 1:1. 
empregando-se uma solução de lauril sulfato de sódio $1 \%$ $(\mathrm{p} / \mathrm{v})$ como meio de liberação (resultados não demonstrados). A diferença nos perfis de liberação da CBZ foi evidenciada neste ensaio, sendo que as partículas preparadas com a adição de PEG 1500, mais porosas, conduziram à liberação de cerca de $30 \%$ a mais de fármaco no mesmo período de tempo, que aquelas obtidas unicamente com $\mathrm{ABC} 70$. Estes resultados fornecem bons indicativos do potencial que a adição de polietilenoglicol como adjuvante farmacêutico nas formulações de microesferas. A possibilidade de controlar o desenvolvimento de poros por meio de estudos de otimização do peso molecular e concentração de PEG permitiria modular a velocidade com que o fármaco é liberado a partir da matriz polimérica. Neste estudo, a adição de PEG 1500 às formulações de microesferas de acetobutirato de celulose pareceu ser mais promissora, uma vez que permite o desenvolvimento de poros nas partículas sem comprometer a eficiência de encapsulação da CBZ e a estrutura esférica das mesmas.

\section{CONCLUSÃO}

Este estudo permitiu avaliar a viabilidade da preparação de microesferas de CBZ, a partir de misturas de ABC70 e PEGs, tendo como parâmetros a morfologia das partículas e os valores de encapsulação do fármaco. Estudos aprofundados de liberação da $\mathrm{CBZ}$ a partir das microesferas preparadas a partir de misturas de $\mathrm{ABC} 70 \mathrm{e}$ PEG 1500 encontram-se em andamento.

\section{ABSTRACT \\ Effect of polyethylene glycols addition in microsphere formulations of cellullose acetate butyrate on efficacy carbamazepine and particles morphology encapsulation}

With the aim of obtaining carbamazepine (CBZ) prolonged release dosage forms, cellulose acetate butyrate (CAB70) microspheres have been prepared by the emulsion/solvent evaporation method. In order to modulate the CBZ release, the addition of polyethylene glycols (PEG) in the internal phase of the emulsion was carried out. When compared with microspheres prepared only from $C A B 70$, the encapsulation efficiency and the CBZ content were drastically reduced by the PEG 4000 addition. On the other hand, the incorporation of $10 \%(w / w)$ of PEG1500 did not affect the drug content in the microspheres. Most porous particles werw observed by SEM when PEG 1500 or 4000 was added to the formulations, however the lost of the spherical shape of the particles was verified when an ABC70:PEG 4000 1:1 mixture was used to prepare the microspheres. The addition of PEGs did not appear to affect the mean diameter of the particles. The decrease of the CBZ content was related to the PEG extraction during the solvent evaporation step and to its ability to dissolve the CBZ in the external phase of the emulsion. The porosity development control, by adjusting the amount or the molecular weight of PEG, can be useful in the drug release from microparticles.

UNITERMS: Carbamazepine. Microspheres. Cellulose acetate butyrate. Polyethylene glycol 1500. Polyethylene glycol 4000. Emulsion/solvent evaporation method.

\section{REFERÊNCIAS BIBLIOGRÁFICAS}

ARNAUD, P., BOUÉ, C., CHAUMEIL, C. Cellulose acetate butyrate microparticles for controlled release of carbamazepine. J. Microencapsulation, v. 13, n. 4, p. 407-417, 1996.

BRADWAJ, S. B., SHUKLA, A. J., COLLINS, C. C. Effect of varying drug loading on particle size distribution and drug release kinetics of verapamil hydrocloride microspheres prepared with cellulose esters. $J$. Microencapsulation, v. 12, n. 1, p. 71 -81, 1995.

BAHL, Y. and SAH, H. Dynamic changes in size distribution of emulsion droplets during ethyl acetate-based microencapsulation process. AAPS Pharm. Sci. Tech., v. 1, n. 1, 2000. Disponível em http://www.pharmascitech. com. Acesso em : 26 março de 2001.

DOSHI, D. H., RAVIS, W. R., BETAGERI. G. V. Carbamazepine and polyethylene glycol solid dispersions: Preparations, in vitro dissolution and characterization. Drug Devel. Ind. Pharm., v. 23, n. 12, p. 1167-1176, 1997.

EASTMAN Cellulose Esters for Pharmaceutical Drug Delivery. Disponível em: http://www.eastman.com. Acesso em: dez. 2000.

KHAWLA, A. A., CONTRERAS, L. G., LU, D. L. Preparation and evaluation of sustained release AZTloaded microspheres: Optimization of the release characteristics using response surface methodology. $J$. Pharm. Sci., v. 85, n. 2, p. 144-149, 1996. 
KHIDR, S. H., NIAZY, . M. el-SAYED, Y. M. Development and in vitro evaluation sustained release meclofenamic acid microspheres. J. Microencapsulation, v. 15, n. 2, p. 153-62, 1998.

KOROLKOVAS,A Dicionário Terapêtico Guanabara. Ed. 1998/1999. Rio de Janeiro: Guanabara Koogan, 1998. p. 1.14 .

REYNOLDS, J. E. F., ed. Martindale. The extra Pharmacopeia. 29 ed. London: The Pharmaceutical Press, 1989. p. 12-21.

UNITED STATES PHARMACOPEA. 17 ed. Rockville, 1997. v.1, p. 166-173, 277-288, 714-720, 1585-1595.

WADE, A, WELLER, P. Handbook of Pharmaceutical Excipientes. 2. ed. London: The Pharmaceutical Press, 1994. p.355-361.

WATTS, P. J., DAVIES, M. C., MELiA, C. D. Microencapsulation using emulsification/solvent evaporation: an overview of techniques and applications. Crit. Rev. Ther. Drug Car. Syst., v.7, n. 3, p. 235-259, 1990.
YEH, M. K., JENKINS, P. G., DAVIS, S.S., COOMBES, A G. A . Improving the delivery capacity of microparticle systems using blends of poly (DL-lactide co-glycolide) and poly (ethylene glycol). J. Controlled Release, v. 37, p. 1-9, 1995.

YEH. M. K. The stability of insulin in biodegradable microparticles based on blends of lactide polymers and polyethylene glycol. J. Microencapsulation, v.17, n. 6, p. 743-756, 2000

YUAN, J., WU, S. Comprimidos de liberação controlada via compressão direta. Um estudo de viabilidade usando acetato de celulose e acetobutirato de celulose. Pharm. Technol. Brasil, v. 5, n. 1, p. 34-42, 2001.

ZERROUK N., CHEMTOB, C., ARNAUD, P., TOSCANI, S., DUGUE, J. In vitro and in vivo evaluation of carbamazepine-PEG 6000 solid dispersions. Int. J. Pharm., v. 225, p. 49-62, 2001.

Recebido para publicação em 14 de dezembro de 2001. 